\title{
Efeito de Impactos Repetidos de Baixa Energia em Compósitos Pultrudados
}

\author{
Willy Ank de Morais, José Roberto M.d'Almeida \\ Departamento de Ciência dos Materiais e Metalurgia, PUC - Rio \\ Leonardo B. Godefroid \\ Departamento de Engenharia Metalúrgica e de Materiais, UFOP
}

\begin{abstract}
Resumo: Danos internos podem ser gerados em compósitos de matriz polimérica submetidos a impactos de baixa energia. $\mathrm{O}$ efeito repetido de eventos desse tipo pode levar, eventualmente, à falha da estrutura. Neste trabalho dois tipos de perfis pultrudados foram submetidos a impactos por queda livre de peso de $0,5 \mathrm{~m}$ e $1,0 \mathrm{~m}$ de altura. Os resultados obtidos foram analisados em função da disposição das fibras nos perfis e mostram que a existência de uma camada rica em resina em um dos perfis, contribui para reduzir significativamente o desempenho deste perfil. Este comportamento foi associado à formação prematura de trincas na camada rica em resina. O número de eventos de impacto, o tempo de duração de cada choque e a forma da área danificada foram correlacionados com a presença, ou não, da região rica em resina. Os resultados obtidos indicam ainda que a diferença na microestrutura dos perfis analisados é mais significativa para os ensaios de menor energia (queda de $0,5 \mathrm{~m}$ ). Para os ensaios de maior energia (queda de $1 \mathrm{~m}$ ) a configuração do material teve apenas uma importância secundária.
\end{abstract}

Palavras-chave: Compósitos pultrudados, impacto de baixa energia, defeitos.

\section{Effects of low energy repetitive impacts in pultruded composites}

\begin{abstract}
Damage can be produced in polymers composite materials submitted to low energy impact events. The cumulative effect of such repetitive impacts could finally cause failure of the structure. In this work two pultruded composites were submitted to drop-weight tests. The results obtained were evaluated according to the fiber distribution in the composites. It was also shown that the presence of a resin rich layer in one of the composites strongly reduces the performance of this composite. This behavior was associated with the development of cracks in the resin rich layer. The number of repeated impacts, the duration of the impact and the shape of the damaged area were correlated to the presence, or the absence, of the resin rich layer. The results obtained also show that the difference between the microstructures of the pultruded profiles analyzed is more significant when low energy impact tests were performed (drop height : $0.5 \mathrm{~m}$ ). For the tests performed with a higher impact energy (drop height : $1 \mathrm{~m}$ ) the microstructure plays only a secondary role.
\end{abstract}

Keywords: Pultruded composites, low energy impact tests, defects

\section{Introdução}

Materiais compósitos de matriz polimérica reforçada por fibras têm, atualmente, um largo emprego industrial ${ }^{[1,2]}$. Dentre as vantagens apresentadas por esses materiais pode-se destacar baixa densidade, $\rho$, e altas resistência mecânica, $\sigma$, e rigidez, $E^{[3]}$.
Assim sendo, esses materiais são largamente empregados nas indústrias automotiva e aeroespacial, onde propriedades específicas elevadas, ou seja altas razões $\sigma / \rho$ e $E / \rho$, são essenciais ${ }^{[3]}$. $\mathrm{Na}$ indústria aeroespacial, em particular, o emprego de compósitos de matriz polimérica abrange desde membros estruturais, como a empenagem vertical de grandes aviões

Autor para correspondência: José Roberto M. d'Almeida, Departamento de Ciências dos Materiais e Metalurgia, Pontífica Universidade Católica do Rio de Janeiro, Rau Marques de São Vicente 225, CEP:22453-900, Rio de Janeiro, RJ. E-mail: dalmeida@dcmm.puc-rio.br. 
comerciais, até estruturas secundárias como painéis de recobrimento das asas dos aviões ${ }^{[1,4-6]}$.

Um problema prático ao qual os compósitos estão sujeitos, e extremamente importante para o desempenho seguro desses materiais nas diversas aplicações em que são usados, está ligado aos danos produzidos por impactos de baixa energia ${ }^{[7-10]}$. Nesses eventos, o compósito pode se apresentar sem qualquer defeito externo e, portanto, eventuais danos estruturais ocasionados pelo impacto podem passar desapercebidos. Um exemplo típico de impactos de baixa energia sobre uma estrutura em compósitos são os pedriscos jogados pelas rodas de um aeroplano sobre sua fuselagem, durante as operações de pouso, decolagem e taxiamento. $\mathrm{O}$ material submetido a repetidos impactos de baixa energia pode vir eventualmente a falhar em serviço, embora seu aspecto externo não esteja seriamente comprometido. Este mesmo problema ocorre em outras aplicações industriais, como por exemplo nas grades que formam boa parte dos pisos das plataformas de petróleo e que estão sujeitas a queda freqüente de ferramentas e de outros objetos mais pesados. Nesta aplicação são usados perfis pultrudados, cujo desempenho em serviço deve ser cuidadosamente avaliado em função da microestrutura de cada perfil.

Assim sendo, a análise dos defeitos gerados em compósitos de matriz polimérica tem grande interesse prático e diversos trabalhos vêm sendo desenvolvidos nesta área. Alguns trabalhos empregam o formalismo da Mecânica de Danos para analisar a evolução do processo de geração de defeitos ${ }^{[11]}$. Este é, entretanto, um enfoque macromecânico que não permite descrever a contribuição relativa dos diversos micromecanismos que podem contribuir para a falha de um compósito, a saber: formação de tricas na matriz, fratura das fibras, ruptura da interface fibra-matriz e delaminações ${ }^{[12]}$. Deste modo, tem-se tentado avaliar, e quantificar, a contribuição destes diversos micromecanismos no processo de falha, seja pela análise das curvas carga vs. tempo, obtidas em ensaios instrumentados de impacto ${ }^{[9,13-15]}$, pela energia residu$\mathrm{a}^{[16,17]}$, ou por métodos de ensaios não destrutivos ${ }^{[18,19]}$.

Neste trabalho é feita a análise do comportamento de perfis pultrudados a impactos de baixa energia. Os perfis são provenientes de duas linhas de pultrusão, apresentando, assim, diferenças na distribuição das camadas de fibras. O acúmulo de defeitos devido ao processo repetitivo de impacto foi avaliado e discutido em função da disposição das fibras no perfil.

\section{Impactos de Baixa Energia}

Os eventos de impacto são normalmente classificados em impactos de alta ou baixa energia, de acordo com o valor da energia cinética inicial do impactador $\left(E_{c}\right)$. Este valor depende da velocidade de impacto e da massa do impactador ( $\mathrm{m})$, de acordo com a equação:

$$
\mathrm{E}_{\mathrm{c}}=1 / 2 \mathrm{~m} \cdot \mathrm{v}^{2}
$$

Na realidade, a velocidade de impacto afeta muito o comportamento de um material, devido à sensibilidade da resposta mecânica à taxa de carregamento ${ }^{[20,21]}$. Por isto, alguns autores preferem classificar os eventos de impacto de acordo com a velocidade do impactador ${ }^{[10,19]}$.

Embora não haja uma definição exata dos valores que distinguem um evento de impacto a baixa energia de um outro a alta energia, os ensaios concebidos para os dois casos são realizados em condições distintas, que deixam claro que tipo de resposta ao impacto está sendo avaliada. De uma maneira geral, em eventos de impacto de baixa energia deseja-se avaliar os defeitos introduzidos no material pelo impacto. Neste caso, parte-se do princípio de que a estrutura necessariamente sobreviverá ao impacto e o problema será determinar qual foi o grau de redução na sobrevida do material. Quando se tratam de impactos de alta energia, o principal interesse está no comprometimento global da estrutura, ou seja, se a mesma conseguirá manter a sua responsabilidade estrutural após o impacto.

Os eventos de impacto a baixa energia são de ocorrência comum nas estruturas de engenharia em geral. No caso de estruturas em compósitos esse tipo de impacto leva à geração de danos internos, que podem comprometer a integridade da estrutura a médio ou longo prazo, principalmente quando são eventos repetitivos. Dentre os eventos característicos de impacto de baixa energia pode-se $\operatorname{citar}^{[22]}$ :

- a incidência de granizo na fuselagem, principalmente nos bordo de ataque, das aeronaves;

- o choque de pequenos pedriscos sobre os "flaps" das aeronaves durante o procedimento de aterrissagem;

- a queda de ferramentas de manutenção sobre estruturas de materiais compósitos;

- esbarrões dos cascos de embarcações contra recifes, rochedos e no cais de atracação.

Em termos de energia, esses impactos implicam em um aporte de até $20 \mathrm{~J}$ nas estruturas impactadas. Entretanto, esse valor pode variar muito de acordo com 
o grau de dano que se pode impor, com as condições de impacto e com a geometria do impactador e da estrutura em uso. As velocidades de impacto dependem da massa do impactador utilizado e, de um modo geral, as velocidades que recaem na condição de impacto de baixa energia variam até $20 \mathrm{~m} / \mathrm{s}$, para uma massa do impactador de até $100 \mathrm{~g}^{[10,22]}$. Deve-se ressaltar, entretanto, que estes valores não são limitantes e a classificação do tipo de impacto depende fundamentalmente das condições de contorno do problema.

\section{Materiais e Métodos}

Foram analisados dois tipos de perfis pultrudados de fibra de vidro e matriz poliéster, que embora tenham a mesma geometria externa apresentam diferenças quanto ao posicionamento das camadas de fibra. Um dos perfis tem fibras dispostas de modo não simétrico em relação à seção reta do pultrudado. Neste perfil, que será denominado perfil I a partir deste ponto, existe uma camada interna de resina separando as camadas de fibra, Figura 1a. No outro perfil, denominado perfil II, as fibras formam uma camada contínua ao longo da espessura, Figura 1b. Ambos os perfis têm camadas externas ricas em resina e um véu de super-

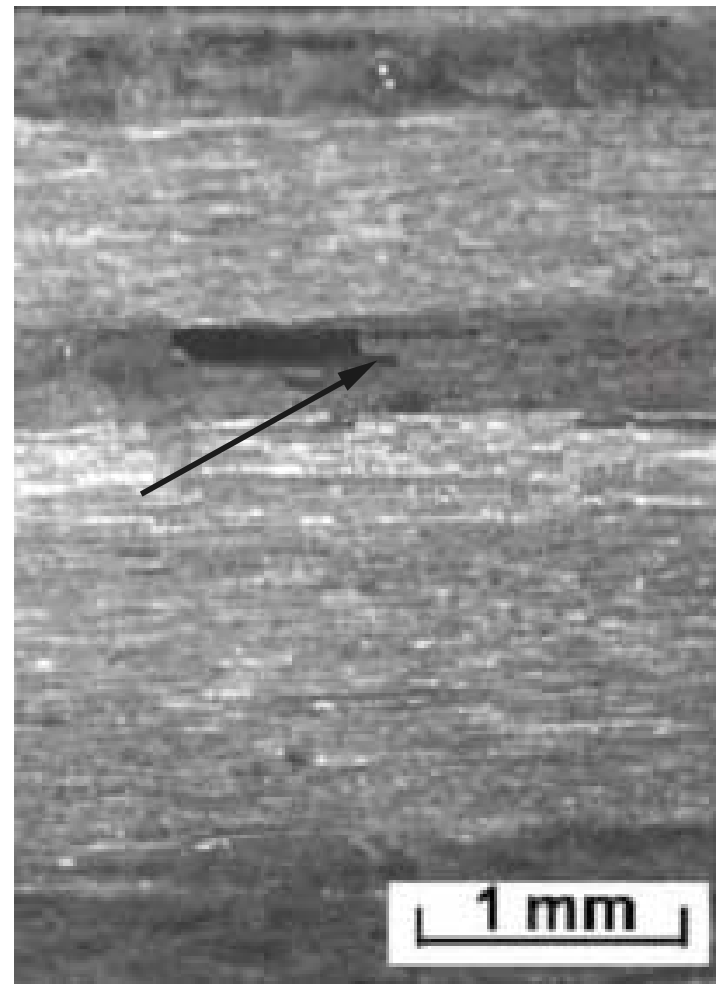

(a) fície, usado para melhorar o acabamento superficial do produto $^{[23]}$. A geometria dos perfis e as dimensões dos corpos-de-prova usados estão mostradas na Figura 2 e na Tabela 1 , respectivamente.

Tabela 1. Dimensões dos corpos de prova. Todas as dimensões em milímetros.

\begin{tabular}{ccccc}
\hline $\mathbf{L}_{\mathbf{1}}$ & $\mathbf{L}_{\mathbf{2}}$ & $\mathbf{L}_{\mathbf{3}}$ & $\mathbf{e}_{\mathbf{1}}$ & $\mathbf{e}_{\mathbf{2}}$ \\
\hline $350 \pm 5^{*}$ & $90 \pm 3,5^{*}$ & $30 \pm 0,3^{*}$ & $5 \pm 0,08$ & $3,3 \pm 0,12$ \\
\hline
\end{tabular}

* desvio padrão relativo ao erro no procedimento de corte das amostras

Para realização dos ensaios foi construído um equipamento de queda de peso, conforme mostrado na Figura 3, que permite a realização de ensaios a velocidades de até $10 \mathrm{~m} / \mathrm{s}$. Este equipamento, cuja descrição detalhada foi feita em um trabalho anterior ${ }^{[24]}$, é formado por quatro sub-sistemas básicos, a saber:

- estrutura rígida onde estão apoiadas e montadas as diversas partes do equipamento;

- sistema magnético de fixação do eletroimã e cabos guia para o impactador;

- impactador dotado de uma célula de carga;

- sistema de aquisição de dados.

A instrumentação do impactador permite obter curvas de carga vs. tempo de duração do choque e, portan-

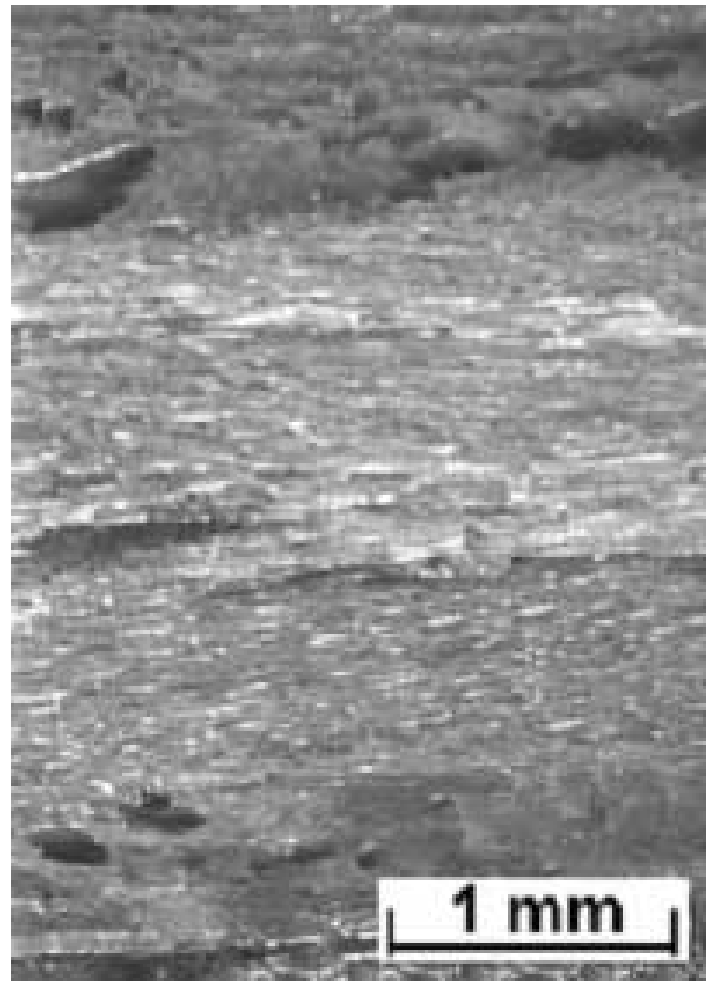

(b)

Figura 1. Disposição das fibras nos perfis. a) Perfil I; b) Perfil II. A seta no perfil I indica a camada interna rica em resina 


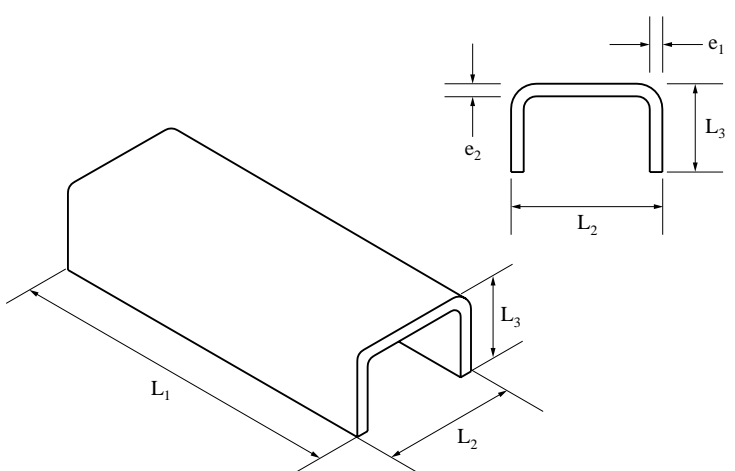

Figura 2. Geometria dos perfis pultrudados.

to, é possível determinar, além da energia absorvida, o tempo de duração do choque e a força e deslocamentos máximos que ocorrem em cada evento de impacto. Deve-se destacar que para impactos de baixa energia é mais importante determinar a evolução dos danos sendo introduzidos no material do que o valor da energia absorvida no impacto, pois não ocorre ruptura do material em um único evento. Assim sendo, os dados obtidos são, comumente, analisados em relação ao número percentual de impactos para a ruptura, ou seja: a razão entre o evento de impacto sendo analisado e o número total de impactos no qual o material falha.

A fim de que a condição de ensaio fosse a mais severa possível, os corpos-de-prova foram engastados à base da máquina por meio de um porta amostra ${ }^{[10]}$.

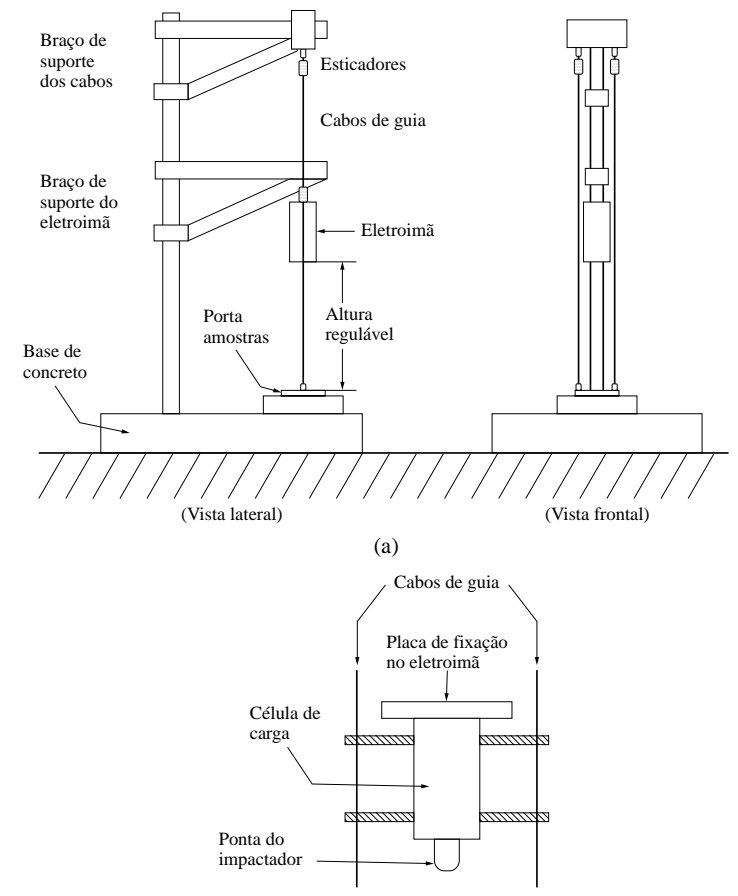

(b)

Figura 3. (a) Representação da máquina de impacto por queda de peso. (b) Detalhe da ponta do eletroimã com célula de carga e o impactador

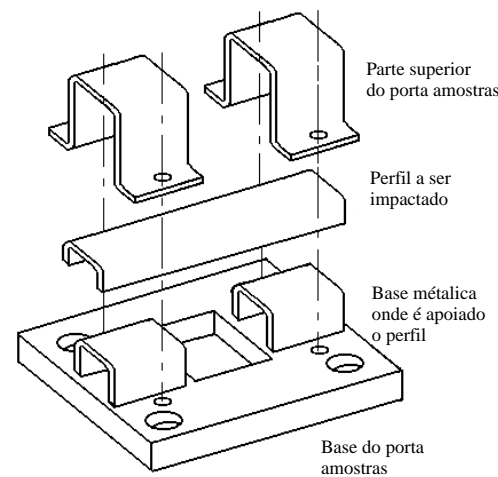

Figura 4. Dispositivo empregado para prender o corpo-de-prova à base da máquina de ensaio.

O dispositivo empregado está mostrado na Figura 4. Para cada tipo de perfil analisado foram testadas três amostras para quedas de peso de $1 \mathrm{~m}$ de altura e duas amostras para queda de peso de $0,5 \mathrm{~m}$. Estes ensaios simulam, respectivamente, eventos de impacto que levam a uma falha rápida ou mais lenta do material em serviço. A massa do impactor usado foi de 765 g. O critério utilizado para definir a falha de uma amostra foi a queda brusca que ocorre na carga suportada pelo compósito quando os danos gerados já atingiram a face posterior ao impacto.

\section{Resultados e Discussão}

A resistência ao impacto dos perfis pultrudados em relação à queda repetitiva de peso está mostrada na Tabela 2. Pode-se observar que o perfil II apresenta melhor desempenho, tanto nos ensaios de curta duração (maior altura de queda) quanto nos ensaios de longa duração (menor altura de queda). Este comportamento foi associado à diferença na disposição das fibras de vidro dentro do perfil. Conforme mostrado na Figura 1, o perfil I tem uma camada interna de resina, separando as camadas ricas em fibra, enquanto o perfil II apresenta camadas ricas em resina apenas nas bordas.

A presença de uma camada interna rica em resina promove o aparecimento precoce de danos internos no material devido, às ondas de choque trativas geradas durante o evento de impacto ${ }^{[21]}$. De fato, a resina poli-

Tabela 2. Número de impactos repetitivos necessários para causar falha dos perfis.

\begin{tabular}{ccc}
\hline Altura da queda de peso, $\mathbf{m}$ & Perfil I & Perfil II \\
\hline 0,5 & $43,5 \pm 6,4$ & $86,5 \pm 30,4$ \\
1,0 & $7,3 \pm 0,6$ & $9,0 \pm 1,0$ \\
\hline
\end{tabular}


éster é um material frágil, apresentando valores de tenacidade à fratura inferiores até aos baixos valores associados aos materiais cerâmicos ${ }^{[25]}$. Assim sendo, nesta camada interna vão sendo geradas, e acumuladas, microtrincas, que contribuem para a falha prematura deste perfil. A origem destas microtrincas ocorre em bolhas e vazios oriundos do processo de fabricação e nas próprias interfaces entre a camada de resina e as camadas ricas em fibras ${ }^{[26]}$. Nestas interfaces existe uma descontinuidade na propagação das ondas de choque e, portanto, pode-se ter diferenças significativas nos campos de tensão e deformação atuantes ${ }^{[21]}$.

$\mathrm{O}$ aspecto geral das curvas carga vs. tempo de duração do choque foi comum para ambos os tipos de perfis analisados. Um conjunto típico dos dados obtidos está mostrado na Figura 5. Pode-se observar que há uma queda na carga suportada pelo material e um aumento do tempo do evento de impacto à medida que o número de impactos aumenta. Este comportamento ocorreu para ambas as alturas de queda analisadas e está diretamente relacionado a geração de defeitos internamente ao material. Os sucessivos impactos causam a redução da rigidez do compósito ${ }^{[1]}$ e o material tem uma deflexão maior para cada novo evento de impacto. O nível de geração de defeitos internos pode, assim, ser avaliado analisando-se o aumento do tempo de duração de cada evento de impacto.

Na Figura 6 estão mostrados os gráficos de duração do impacto em função do número percentual de impactos. Pode-se observar que o comportamento global obtido para os perfis e energias de impacto analisadas foi o mesmo. Nos primeiros impactos o tempo de duração aumenta pouco. Após cerca de $20 \%$ da vida do material a derivada da curva torna-se positiva e a duração do tempo

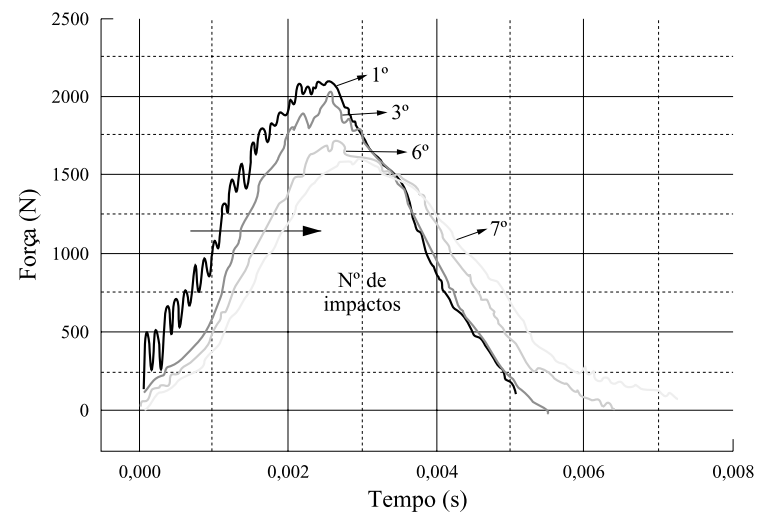

Figura 5. Aspecto comum das curvas carga vs. tempo de duração do choque dos perfis testados. Exemplo do comportamento do perfil II, queda do peso de $1 \mathrm{~m}$. A seta $(\rightarrow)$ indica a ordem crescente do número de impactos. de impacto cresce a uma taxa constante. Os resultados obtidos para os impactos de $0,5 \mathrm{~m}$ de altura corroboram a análise que indica que os perfis do tipo II tem um melhor desempenho. Os gráficos das Figuras $6 \mathrm{a}$ e $6 \mathrm{~b}$ mostram que após $40 \%$ de vida o tempo de duração do impacto é maior para os compósitos do tipo I. Ou seja, existe um acúmulo maior de danos neste material em relação ao perfil II, conforme esperado pela análise dos dados mostrados na Tabela 2. Os resultados obtidos para os impactos de $1 \mathrm{~m}$ de altura não mostraram este comportamento. Entretanto, devido ao maior nível de energia e a pequena diferença entre o número de impactos que causam a falha do material sob estas condições de ensaio, pode-se esperar que os danos produzidos sejam muito mais severos. Assim sendo, os resultados obtidos indicam que a diferença na configuração do material tem uma importância apenas secundária na resposta mecânica dos perfis sob maior nível de energia de impacto. De fato, conforme mostrado na Figura 7, pode-se observar que a energia média dissipada em cada evento de impacto com queda de $1 \mathrm{~m}$ foi, aproximadamente, o dobro da energia dissipada nas quedas de $0,5 \mathrm{~m}$.

Além disto, devido à diferença entre os níveis de energia, a interação entre o impactador e a amostra é diferente. Nos ensaios realizados com queda de $0,5 \mathrm{~m}$ as contribuições devido à flexão do perfil e à delaminação, com geração de trincas nas interfaces fibra-matriz e formação de trincas na matriz, são maximizadas, enquanto no ensaio com queda de $1 \mathrm{~m}$ a resistência do compósito à penetração é mais importante ${ }^{[13]}$. A presença de uma camada interna rica em resina é, assim, mais relevante para os ensaios de longa duração, conforme indicam os resultados experimentais obtidos.

$\mathrm{O}$ aspecto macroscópico da fratura dos perfis pultrudados está mostrado na Figura 8. Pode-se observar que as áreas danificadas na superfície frontal ao impacto são semelhantes, independendo do tipo de perfil e da altura do impacto. A geometria em forma de calota esférica observada foi associada à deformação homogênea da camada superficial dos perfis em contato com o impactador ${ }^{[27,28]}$.

As áreas danificadas na superfície posterior variaram em função do tipo de perfil. No perfil II a falha ficou restrita a área sob o impactador. O tipo de falha observado representa uma ruptura localizada, devido ao acúmulo de defeitos sob a área de impacto. A forma observada para a falha é comum a apresentada por compósitos reforçados por tecidos ${ }^{[29]}$, e é devida ao véu de superfície presente nos compósitos. Para os 


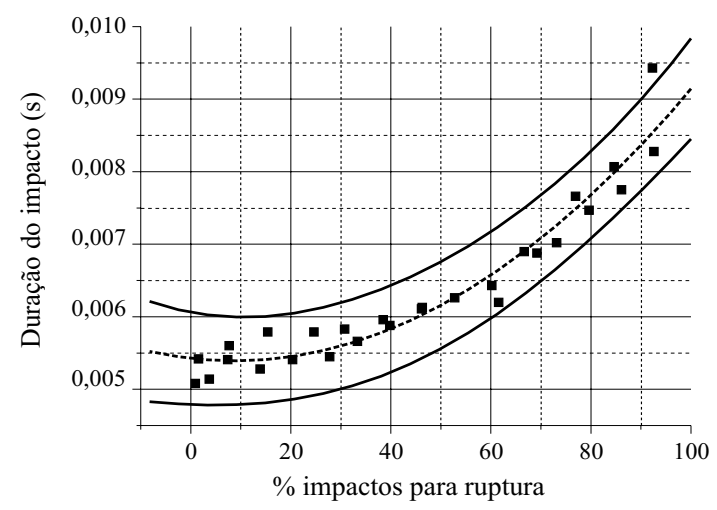

(a)

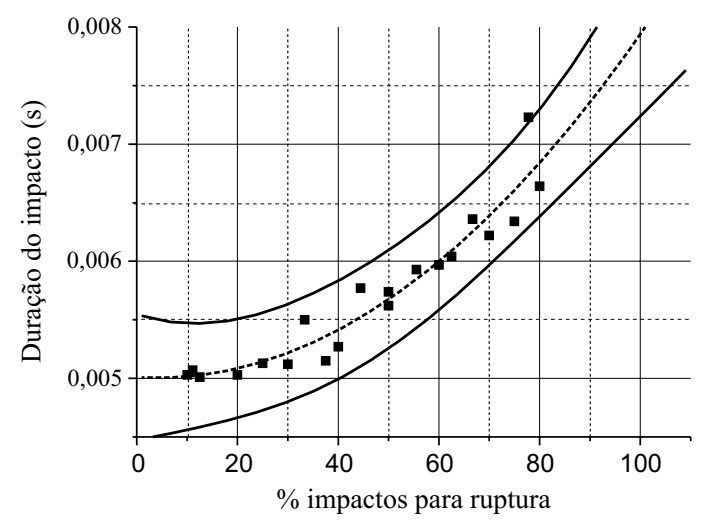

(c)

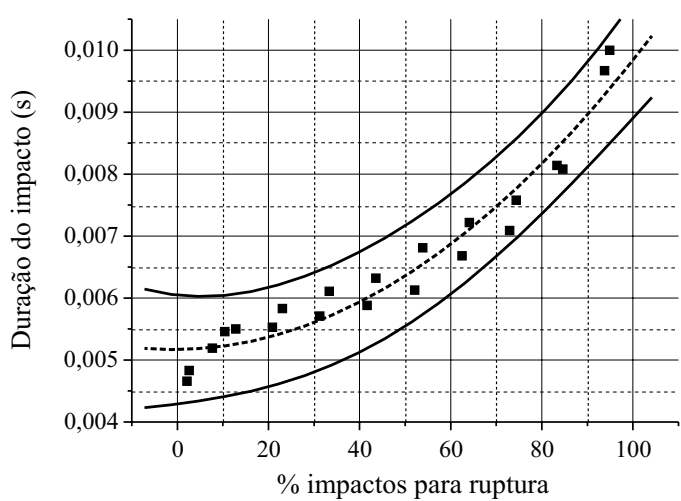

(b)

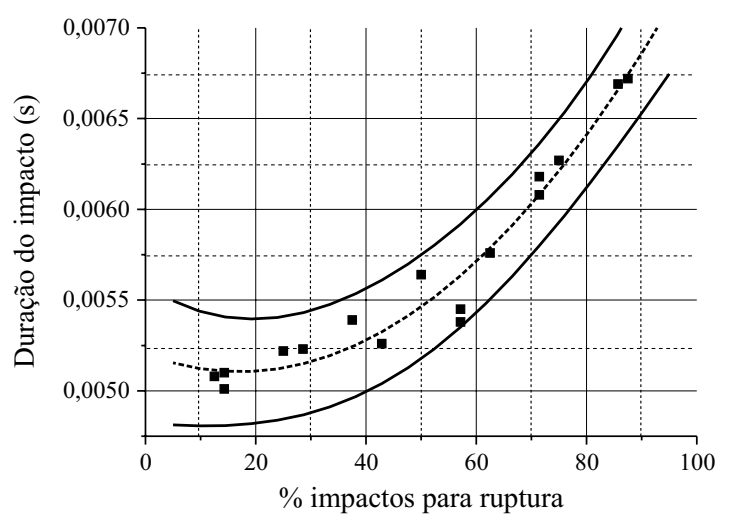

(d)

Figura 6. Evolução da duração do tempo de impacto em função do percentual do número de impactos. a) Perfil II, 0,5 m; b) Perfil I, 0,5 m; c) Perfil II, $1 \mathrm{~m}$; d) Perfil I, $1 \mathrm{~m}$.

perfis do tipo I observa-se uma falha alongada na direção longitudinal do perfil, ou seja, paralelamente à direção das fibras. Este tipo de falha é semelhante ao observado para laminados unidirecionais ${ }^{[17,22]}$ e pode ser atribuído a formação de trincas na camada de resina existente nestes perfis, o que induz ao espalhamento da área de dano para além da região sob o impactador. Falhas deste tipo têm sido atribuídas a um comportamento não isotrópico do material sendo testado ${ }^{[30]}$. Nos perfis testados, o direcionamento da fratura longitudinalmente ao perfil ocorre, ainda, porque nesta direção existe uma menor restrição à deformação, já que as

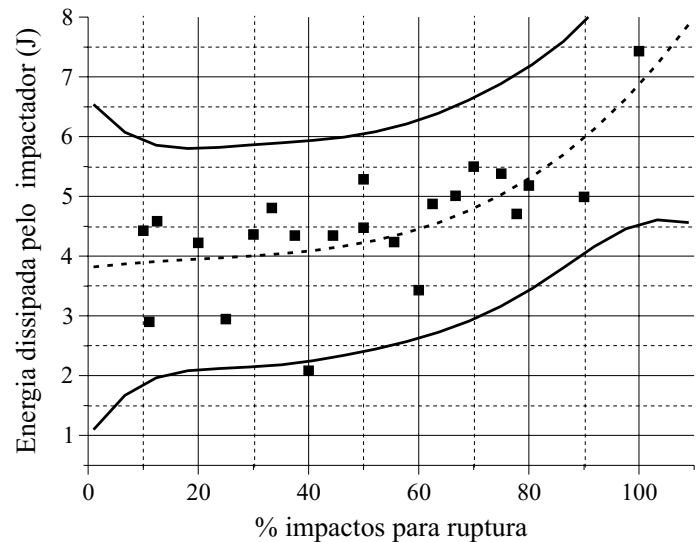

(a)

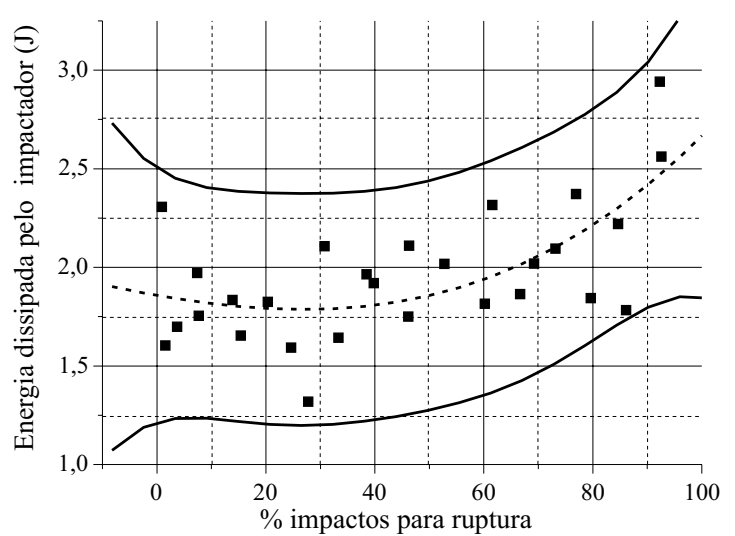

(b)

Figura 7. Valores médios da energia dissipada em cada evento de impacto. a) queda de $1 \mathrm{~m}$; b) queda de $0,5 \mathrm{~m}$. 


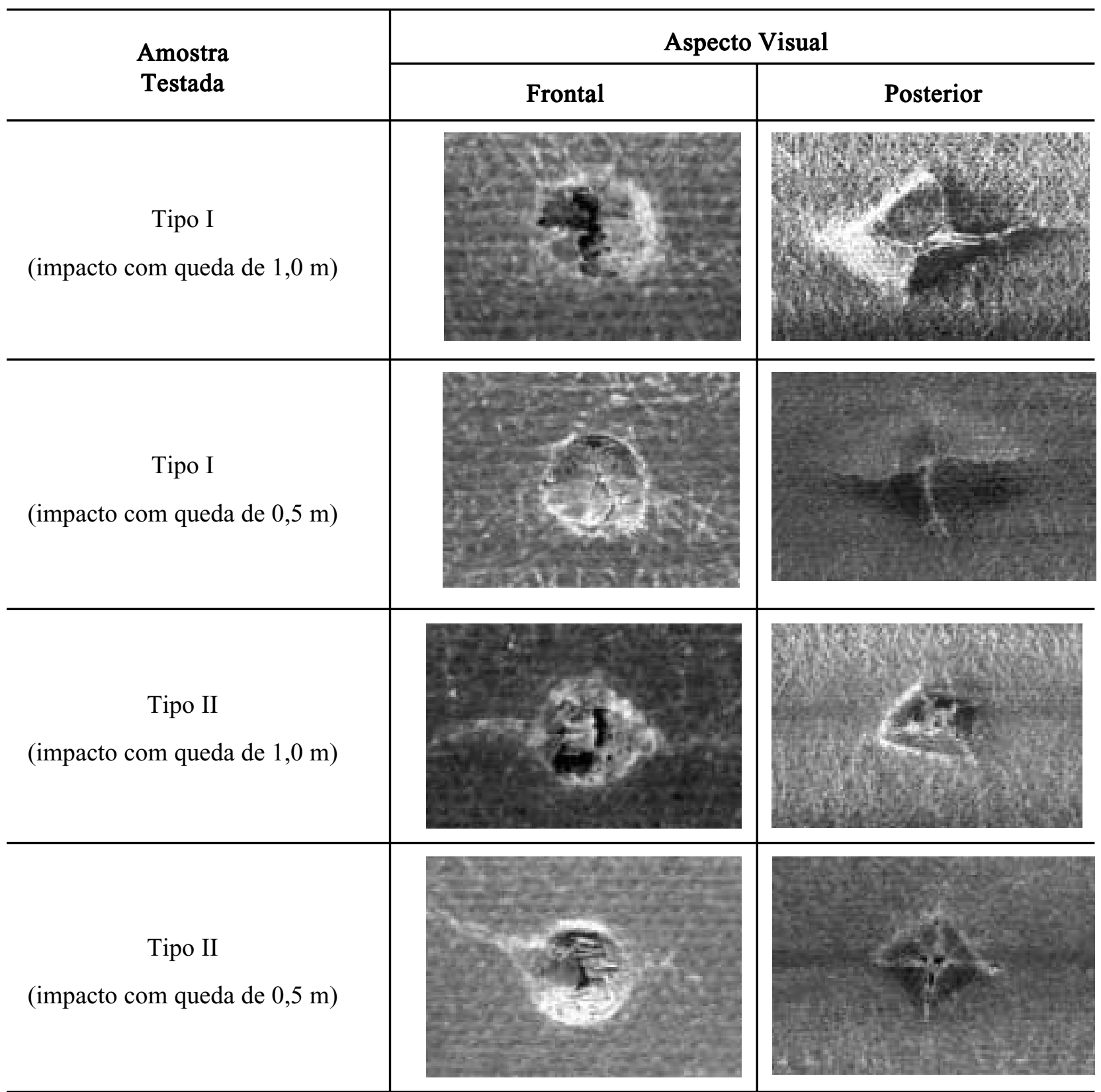

Figura 8. Aspectos do dano causado no ponto de impacto dos perfis pultrudados.

laterais do perfil possuem uma maior restrição geométrica à deformação (Figuras 2 e 4).

\section{Conclusões}

Os resultados obtidos neste trabalho mostram que o desempenho dos perfis pultrudados a impactos de baixa energia é fortemente dependente da microestrutura do compósito. A presença de uma camada interna rica em resina contribui para a queda do número de eventos de impacto que causam a falha do material. Este efeito é mais marcante para os impactos de menor energia, onde o mecanismo de delaminação é mais importante. $\mathrm{O}$ acúmulo de danos no perfil I, que tem uma camada interna rica em resina, foi maior, conforme indicaram o aumento no tempo de impacto e a área danificada sob o impactador. O perfil II apresentou geração de defeitos mais concentrada sob a área do impactador, refletindo uma maior homogeneidade da sua estrutura.

\section{Agradecimentos}

Os autores agradecem à ENMAC Ltda pela doação dos perfis estudados. 


\section{Referências Bibliográficas}

1. Gibson, R. F. - Principles of Composite Material Mechanics, McGraw-Hill, New York (1994).

2. Quinvilan, J. T. - Chairman Section 12: Aplications and Experience in Engineered Materials Handbook, vol.1 - Composites, ASM International, Metals Park, pp.799-848 (1987).

3. Ashby, M. F. - Mater.Sci.\&Technol., 5, pp.517-525 (1989).

4. Anglin, J. M. - in Engineered Materials Handbook, vol.1 - Composites, ASM International, Metals Park, pp.801-809 (1987).

5. James, A. M.; Vaughn, R. L. - Composites, 6, pp.73-80 (1976).

6. McCarty, J. E. - Composites, 24, pp.361-365 (1993).

7. Wu, H. Y. T.; Springer, G. S. - J. of Comp. Materials, 22, pp.518-532 (1988).

8. Choi, H. Y.; Chang, F. K. - J. of Comp. Materials, 26, pp.2134-2169 (1992).

9. Jang, B. P.; Kowbel, W.; Jang, B. Z. - Comp. Sci. \& Technol., 44, 107-118 (1992).

10. Bibo, G. A.; Hogg, P. J. - J. Mater. Science, 31, pp.1115-1137 (1996).

11. Know, Y. W.; Berner, J. - Eng.Frac.Mech., 48, pp.811817 (1994).

12. Hull, D. - An Introduction to Composite Materials, Cambridge UP, Cambridge (1985).

13. Matthews, F. L.; Rawlings, Rawlings, R. D. - Composite Materials: Engineering and Science. Chapman \& Hall, London (1994).

14. Wardle, M. W.; Tokarsky, E. W. - Comp. Tech. Review, 5, pp.4-10 (1983).

15. Sjöblom, P. O.; Hartness, J. T.; Cordell, T. M. J. Comp. Materials, 22, pp.30-52 (1988).
16. Lal, K. M. - Comp.Tech.Review, 6, pp.112-117 (1984).

17. Reed, P. E.; Turner, S. - Composites, 19, pp.193-203 (1988).

18. Hofer, B. - Composites, 18, pp.309-316 (1987).

19. Liu, D. - J. of Comp. Materials, 22, pp.674-692 (1988).

20. Agbossou, Cohen, A.; I.; Muller, D. - Eng. Frac. Mech., 52, pp.923-934 (1995).

21. Meyers, M. A. - Dynamic Behavior of Materials, John Wiley and Sons, New York (1994).

22. de Morais, W. A. - Dissertação de Mestrado, Departamento de Ciência dos Materiais e Metalurgia, Pontifícia Universidade Católica do Rio de Janeiro (1999).

23. de Morais, W. A.; Martins Filho, J. A.; Godefroid, L. B.; d'Almeida, J. R. M. - Anais do 53ํㅡㄹ Congresso da Anual da ABM, Belo Horizonte, 13 a 17 de setembro de 1998, CD-Rom, pp.1149-1163.

24. de Morais, W. A.; Gomes, P. T. V.; Godefroid, L. B.; d'Almeida, J. R. M. - Anais do 53ํㅡㄹ Congresso da Anual da ABM, Belo Horizonte, 13 a 17 de setembro de 1998, CD-Rom, pp.1223-1237.

25. Williams, J. G. - Fracture Mechanics of Polymers, Ellis Horwood Ltd, Chichester (1987).

26. Purslow, D. - Composites, 17, pp.289-303 (1986).

27. Goldsmith, W. - Impact: the theory and physical behaviour of colliding solids, Edward Arnold Publishers, London (1960).

28. Tan, T. M.; Sun, C. T. - J. of Applied Mechanics, 52, pp.6-12 (1985).

29. Park, R.; Jang, J. - J. Applied Polymer Science, 75, pp.952-959 (2000).

30. Christoforou, A. P. - Composite Structures, 26, pp.7782 (1993).

Recebido: 05/12/00

Aprovado: 15/02/01 